\title{
The Effect of Faults on Sweep Efficiency of Secondary Recovery*
}

\author{
by Hikaru Saito**
}

\begin{abstract}
Summary: The study was made on the effect of faults of partially separating flow field between injection and producing wells on sweep efficiency of water flooding recovery with the aid of a potentiometric model. The sweep efficiency depends on the length, position, orientation of the faulting system with respect to the direction of the flood, and ratio of the permeability of the fault to that of the medium.

It was found that the sweep efficiency may be reduced to one half or less when the impermeable faults, whose lengths exceed $7 / 8$ of the well spacing, extend perpendicular to the direction of the flood. For constant fault length, for faulting perpendicular to the flooding direction, the sweep efficiency is $a$ minimum at $b=0.3 d$, where $b$ is the distance between faults and injection well, and $d$ is the spacing from the injection well to the producing well. Where faults are at angles to the direction of flood, the sweep efficiency is a minimum at $\theta=(1 / 3) \pi$, where $\theta$ is the angle between the fault and the flood direction.

The sweep efficiency for a semi-permeable faulting system is larger than that for an impermeable faulting system at a constant fault length, and less than that for a non-faulted system. The longer the fault, the greater would be the effect of permeability ratio on sweep efficiency.

Where injection and producing wells must be partly separated by faults, a greater sweep efficiency will be obtained when the injection well is located such that the area between the injection well and the fault is greater than the area between the producing well and the fault.
\end{abstract}

\section{Introduction}

The well pattern or the distribution of injection and producing wells for a water flooding program is one of the most important factors controlling recovery efficiency. Conventional patterns in a sufficiently large continuous area involved in the secondary recovery program, have been studied by analytical or electric model treatment and are reported by many authorities ${ }^{1)}, 2$. However actual oil reservoirs are confined in geological structures and have geological variations in themselves. The existence of faults in the patterns often reduces the efficiency and, possibly, the applicability of secondary recovery. Interference of conventional flow pattern by faults is inevitable, but modified patterns for such conditions might be developed. Such information is required, if the maximum efficiency is to be obtained.

* Received November 7, 1959.

** Teikoku Oil Company, Ltd., Hirakawa-cho, Chiyoda-ku, Tokyo.
The purpose of this study is to obtain quantitative estimates of the effects on the areal sweep efficiencies of flooding patterns. Some faults are vertical and others are inclined to the bedding planes; fault planes may be impermeable, others semi-permeable and sometimes more permeable than the surroundings. Sometimes faults separate injection and producing wells completely and other partially separate them by socalled "hinge faults"*1. Here, simplifying many complicated factors, studies have been made of two-dimensional faults vertical to the horizontal bedding planes of the flow field. Sweep efficiencies for various faulting systems of partially separating injection and producing wells were obtained with the aid of a potentiometric model.

The "sweep efficiency" is defined as the

*1 The similar condition of a faulting system, which is separating injection and producing wells partially, is known in Wilmington oil field where water flooding is being carried out. The effect of faults on sweep efficiency was considered for the plan there. This thesis pursues basic development on the matter. 
fraction of the volume of oil pay drained by the well that is swept out by the time the water first reaches the well. In the two dimensional analogue, sweep efficiencies represent the fractions of the total area of the pattern or network element that are invaded by the injection fluid by the time it first reaches the producing well $^{3), 4)}$.

\section{Electric Model}

A. The Theory of Electric model

1. Flow of Electricity and its Similarity to Fluid Flow

The theory of electrical models and its application to the flow of fluids in porous media is reviewed by Horner and Bruce ${ }^{23}$.

For flow of electricity, Ohm's low, is;

$$
I=-\frac{1}{\rho} \frac{\partial E}{\partial x}
$$

where $I$; the current flowing through unit area of cross section

$$
\begin{gathered}
\rho ; \text { specific resistivity } \\
\frac{\partial E}{\partial x} ; \text { voltage gradient }
\end{gathered}
$$

The law of continuity expressed for the flow of electricity is

$$
\frac{\partial I}{\partial x}+\frac{\partial I}{\partial y}+\frac{\partial I}{\partial z}=0
$$

Therefore from (2-1) and (2-2), the following equation is obtained.

$$
\frac{\partial^{2} E}{\partial x^{2}}+\frac{\partial^{2} E}{\partial y^{2}}+\frac{\partial^{2} E}{\partial z^{2}}=0
$$

For two-dimensional flow of electricity, (2-3) becomes;

$$
\frac{\partial^{2} E}{\partial x^{2}}+\frac{\partial^{2} E}{\partial y^{2}}=0
$$

For horizontal fluid flow Darcy's law is

$$
q_{x}=-\frac{k_{x}}{\mu} \frac{\partial p}{\partial x}
$$

where $q_{x}$; macroscopic velocity in the $x$-direction

$k_{x}$; permeability in the $x$-direction.

$\mu$; viscosity of fluid

$\frac{\partial D}{\partial x}$; pressure gradient

For horizontal flow of incompressible fluids through homogeneous isotropic porous media, the pressure distribution can be expressed by $^{7), 9)}$;

$$
\frac{\partial^{2} p}{\partial x^{2}}+\frac{\partial^{2} p}{\partial y^{2}}=0
$$

These two equations, (2-4) and (2-6), are completely analogous. Fluid flow is directly comparable to current flow, and electric potential is directly comparable to pressure.

2. Movement of Fluid in a Horizontal Flow
Field

From equations (2-4) and (2-6) the electric model can indicate isopressure lines from isoelectric-potential lines, and a comparison of equation (2-1) and (2-5) shows how current has a relation to volume rate of fluid flow. It is possible to plot the progress of indentified particle in the fluid as it moves from one part of the reservoir to another ${ }^{2), 61}$.

For the horizontal fluid flow along the flow tube through homogeneous isotropic pcrous media, Darcy's law can be written by

$$
q_{s}=-\frac{k}{\mu}-\frac{\Delta p}{d s}
$$

where $q_{s}$; macroscopic velocity along the flow tube

$$
\begin{aligned}
& \frac{\Delta p}{\Delta s} ; \text { pressure gradient along the flow } \\
& \text { tube }
\end{aligned}
$$

Macroscopic velocity along the flow tube, $q_{s}$ is

$$
q_{s}=-\frac{\Delta Q / a}{\Delta t}
$$

where $\Delta Q$; fluid volume of flow through flow tube for time $d t$

$a$; sectional area of flow tube

$J t$; infinitesimal time of flow

Further $\Delta Q$ can be expressed as follows,

$$
J Q=f a \cdot d s
$$

where $f$; fractional porosity

Is ; infinitesimal length of flow tube

The combination of equations, $(2-7),(2-8)$, and (2-9) becomes,

$$
\Delta t=\frac{\mu f}{k} \cdot \frac{\Delta s}{|\Delta p|}
$$

Flowlines emerging from one electrode and going to the other represent the paths along the electric charges or particles of fluid travel from an injection well to a producing well. The advance of a particle along the flowline is shown by $(2-10)$.

Integrating $(2-10)$

$$
t=\frac{\mu f}{k} \int_{0}^{s} \frac{d s}{\left|\frac{\partial p}{\partial s}\right|}
$$

where $s$; length of flowline

$$
t \text {; time required for travel of the }
$$

The positions of marked particles in this system at any subsequent time after emergence from an electrode can be determined by graphical or numerical integration of equation 11) ${ }^{1,2), 5)}$.

\section{B. Apparatus and Measurement \\ 1. Apparatus}

The electrolyte reservoir was made of lucite 
and was $7 \mathrm{in}$. wide by $9 \mathrm{in}$. long by $1 \mathrm{in}$. deep, as shown on Fig. 1. The liquid depth was constant corresponding to a constant permeability $\times$ zone thickness product from the injection to the producing wells. This electrolyte reservoir corresponded to a unit in the linedrive pattern shown in Fig. 2 . Using $\# 26$ plat- inum wire $(0.01594$ in. diameter $)$ for electrodes, the relations between well-bore sizes and well spacings represented are given in Table 1.

The same electrolyte reservoir was used to analyze the case representing edgewater drive to wells in a line, as shown by Fig. 3 , by using a thin copper strip and a platinum wire as
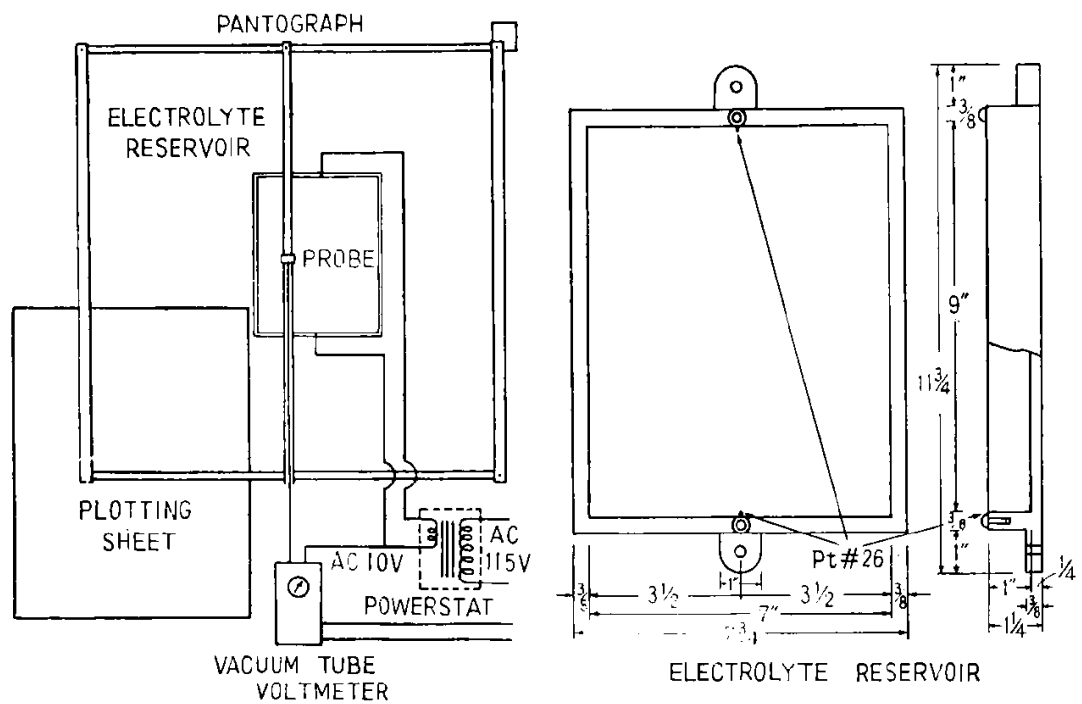

Fig. 1 Model study apparatus

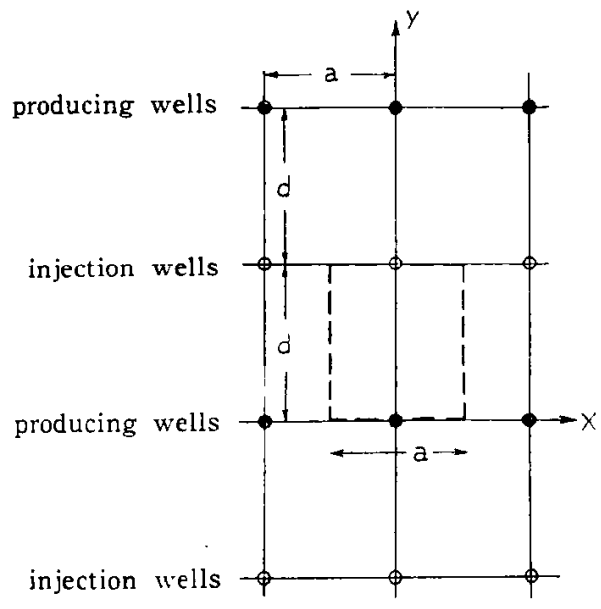

Fig. 2 A diagrammatic representation of a directline-drive well network. Dashed segment represents basic symmetry element.

Table 1. Equivalent geometrical well spacings to the spacing of electrodes of electrolyte reservoir used in this study

\begin{tabular}{c|c|c}
\hline $\begin{array}{c}\text { Size of well } \\
\text { bore hole } \\
\text { diameter }\end{array}$ & Well separation & $\begin{array}{c}\text { Distance between the } \\
\text { lines of injection and } \\
\text { producing wells } \\
\text { (in.) }\end{array}$ \\
\hdashline 3 & $\begin{array}{c}a \\
\text { (ft) }\end{array}$ & (ft) \\
\hline 4 & 110 & 141 \\
5 & 146 & 188 \\
6 & 183 & 235 \\
8 & 219 & 282 \\
& 292 & 376 \\
\hline
\end{tabular}

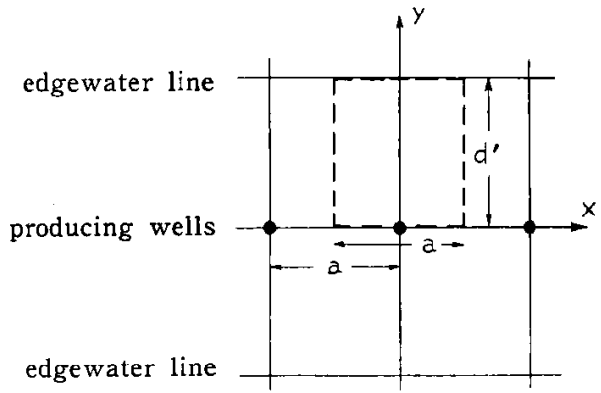

Fig. 3 Edgewater drive to wells in a line

electrodes.

Faults were represented in the model by $1 / 4$ in. thick strips of lucite sealed to the bottom and the wall of the electrolyte reservoir in order to set up nonconductive barriers for electric current. All fault positions studied are illustrated by Fig. 4. A brine of 1 normal $\mathrm{NaCl}$ was used as the electrolyte.

2. Measurement

Ten volts of alternating current were charged between electrodes of source and sink. Measured potential points were plotted directly on a double-size plotting sheet by use of a panto$\mathrm{graph}^{8)}{ }^{10)}$. Isopotential lines, at intervals of 0.2 volt, were constructed through the field except in the vicinity of electrodes where potential gradients were large and large inter- 
(1) Line Drive

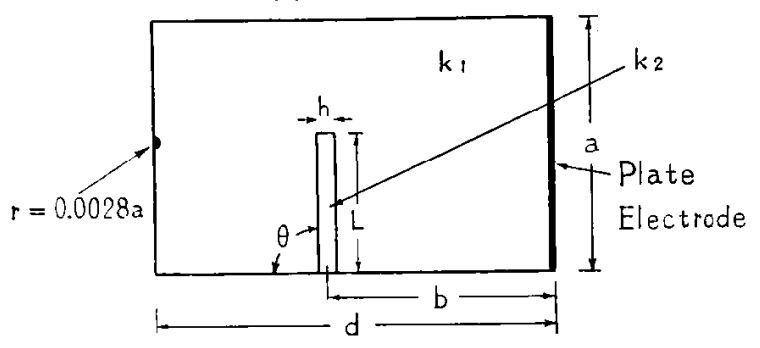

$d=9 \mathrm{a} / 7, \quad h=\mathrm{a} / 28, \quad \theta=\pi / 2, \quad b=d / 2$ conductivity ratio $k_{2} / k_{1}=0$
(a) $L=0$,
(b) $L=0.3 \mathrm{a}$

(c) $L=0.585 \mathrm{a}$,

(d) $L=0.729$ a

(2) Direct Line Drive

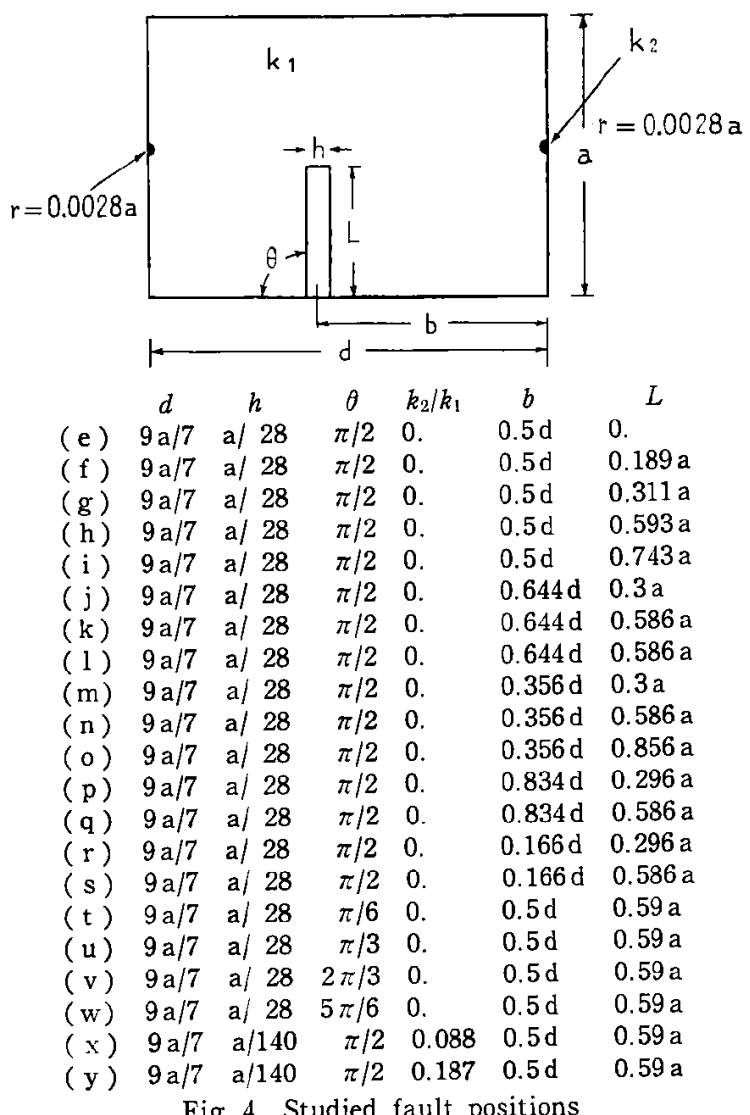

Fig. 4 Studied fault positions

vals used. Flowlines starting from one electrode and going to the other were drawn perpendicular to each isopotential line.

\section{Calculation of Sweep Efficiencies}

As stated in Chapter II, integration of equation (2-11)

$$
t={ }_{k}^{\mu f} \int_{0}^{s}: \frac{d s}{\partial p}
$$

evaluated along the various flowlines in the system, for different valves of $s$, gives the loci of those values of $s$ for which $t=$ constant and will represent the fluid front at the corresponding values of $t$. Equation (2-11) can be integrated graphically, but in this study integrations were made by a numerical method which is expressed by $(3-1)$.

$$
t=\frac{\mu f}{k} \sum_{i}^{n} \mid \begin{gathered}
(J s)^{2} \\
\Delta p
\end{gathered}
$$

where $J p$; potential difference between iospotential lines

Is ; flowline segment cut by isopotentiol lines

$n$; number of isopotential lines spacings between the source and the particular point

Equation (3-1) is not exactly the same as equation (2-11), but by taking $\Delta p$ and $\Delta s$ small, a good approximation was obtained.

For comparison both numerical and graphical integration methods were employed to calculate sweep efficiencies of the steady state flow from a line drive to a producing well. The results were further checked by an analytical solution for this case.

\section{A. Numerical Integration Method}

The calculation for sweep efficiency of a line drive to a producing well, whose potential distribution is illustrated by Fig. 5 is as follows.

For each flowline, $\psi_{1}, \psi_{2}, \psi_{3}, \psi_{5}$ and $\psi_{8},: \frac{(\Delta s)^{2}}{\Delta p}$ was calculated for each interval between isopotential lines. In equation (3-1) $\mu f / k$ is reducible to unit for calculation of sweep efficiency, as it is constant for all flowlines. Letting $\mu f / k=1, t$ is obtained as the summation of all terms of $\frac{(J s)^{2}}{J p}$ from the source to the particular point. The position of the front on flowlines, $\psi_{1}, \psi_{2}, \psi_{3}$, and $\psi_{5}$ are $s=14.10 \mathrm{in} ., 14.24$ in., $14.56 \mathrm{in}$., and $15.63 \mathrm{in}$. respectively at time $t=47.49$ when the front on the shortest-traveltime flowline $\psi_{8}$ first reaches the electrode. Plotting these locations on the map, Fig. 5, the position of the front at $t=47.49$ is found. A sweep efficiency of $83.2 \%$ for this case is obtained by measuring the area behind the front with a planimeter and dividing it by the total area of the network element.

The same procedure was used to find a sweep efficiency of $64.8 \%$ for a direct-line drive well network.

\section{B. Graphical Integration Method}

From Fig. 5, potential values were plotted as functions of flowline length for $\psi_{1}, \psi_{2}, \psi_{3}, \psi_{5}$, and $\psi_{8}$ as shown in Fig. 6. Slopes of those curves, $d \phi / d s$, were measured. Reciprocals of absolute slopes, $1 /(d \phi / d s)$, were then plotted as 


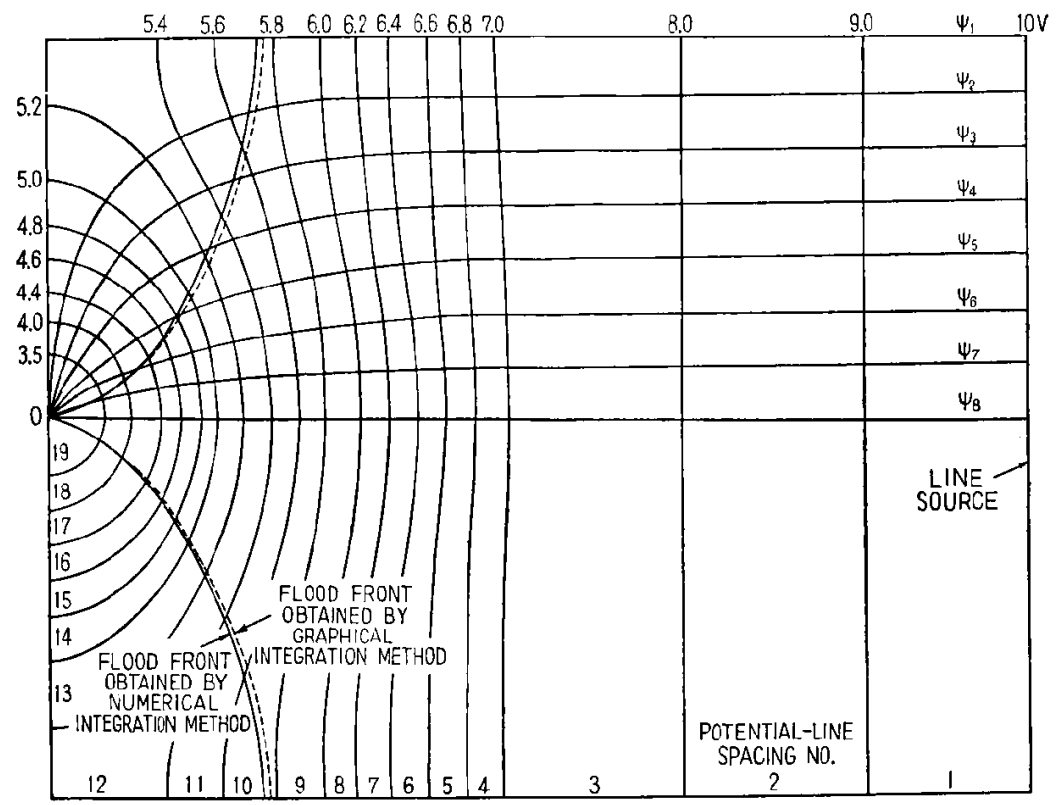

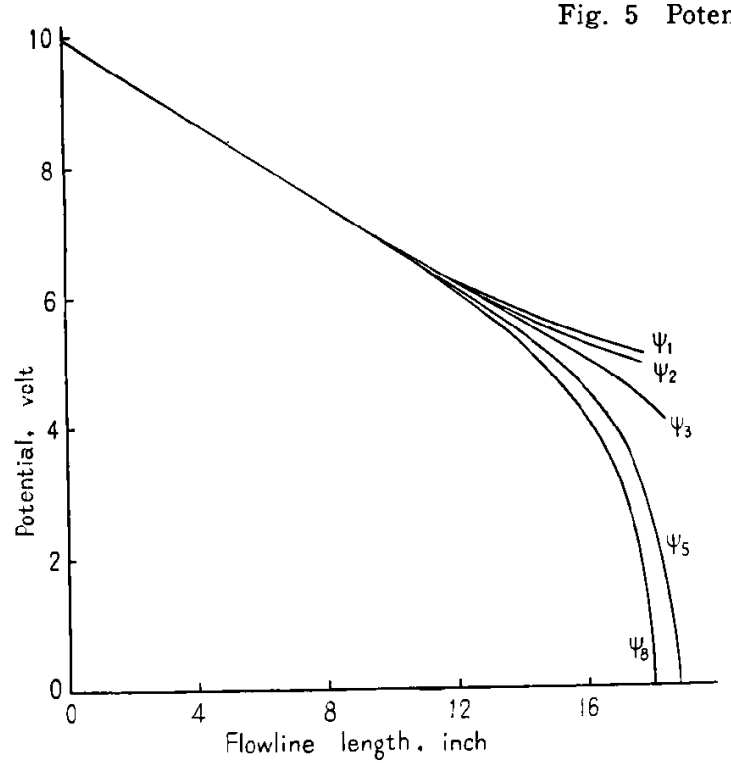

Fig. 6 Potential vs Flowline length

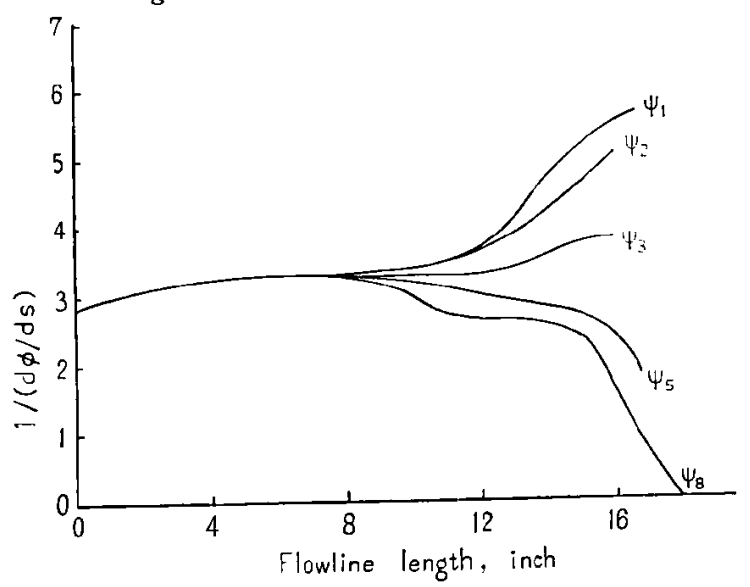

Fig. $71 /(d \varphi / d s)$ vs Flowline length

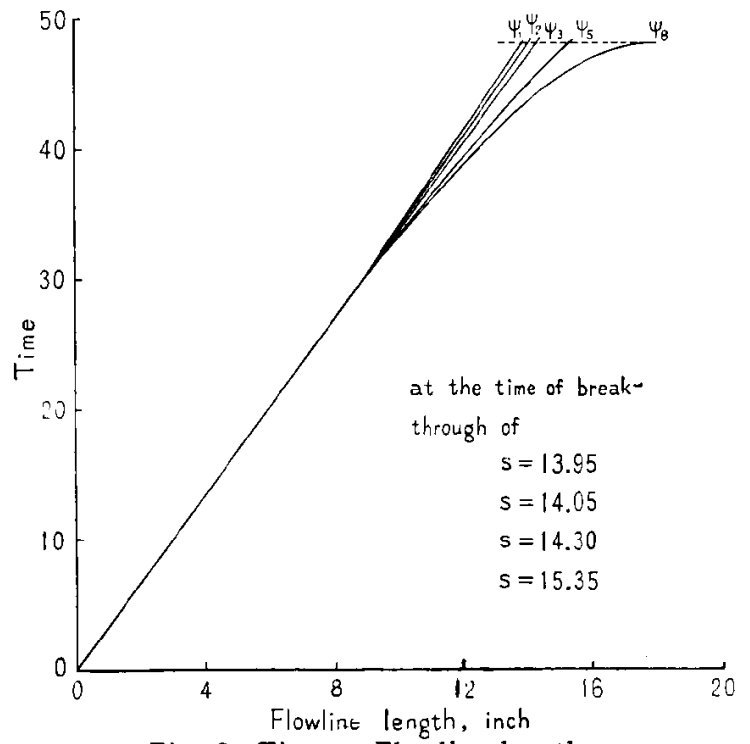

Fig. 8 Time $v$ s Flowline length

shown in Fig. 7. The graphical integration,

$$
\int_{0}^{s} \begin{gathered}
1 \\
d \phi \\
d s
\end{gathered} d s
$$

was made for each flowline as shown in Fig. 8 . At $t=48.0$, the time of breakthrough of $\psi_{8}$, the shortest travel-time flowline, flood fronts $\psi_{1}$, $\psi_{2}, \psi_{3}$, and $\psi_{5}$ were found at $s=13.95,14.05$, 14.30 and 15.35 , respectively. Plotting those on Fig. 5 , a sweep efficiency of $83.0 \%$ was obtained by planimetering.

C. Analytical Method

1. A Direct Line Drive

The pressure distribution of a direct line drive well network, shown by Fig. 2 . is pre- 
sented by Muskat ${ }^{1}$, as follows;

$$
\begin{aligned}
& p(x, y)=\frac{\mu Q}{4 \pi k h}\left[\ln \left(\cosh ^{2 \pi y} \begin{array}{c}
a \\
a
\end{array}-\cos \begin{array}{c}
2 \pi x \\
a
\end{array}\right)\right.
\end{aligned}
$$

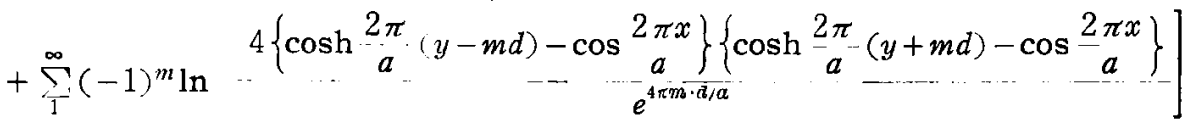

where $p(x, y)$; pressure at the point $(x, y)$

$\mu$; viscosity of fluid

$k$; permeability

$h ;$ thickness porous medium

$a$; spacing of well array

The volume of the injected fluid at time $t$ will be $Q t$ per well, and it will occupy an area $Q t / h f$ in the reservoir. If the network element area associated with each injection well be $A$, the sweep efficiency $E$ will be ${ }^{\text {l) }}$

$$
E=\begin{gathered}
Q t \\
h f \\
A
\end{gathered}=\begin{gathered}
Q t \\
h f A
\end{gathered}
$$

Substituting (2-11) into this equation

$$
E={ }_{k h A}^{\mu Q} \int_{0}^{s} \mid \begin{aligned}
& d p \\
& d s
\end{aligned}
$$

For a direct line drive well network, the shortest-travel-time flowline is that along the $y$-axis from 0 to $d$. Then from (3-2)

$$
\begin{aligned}
\frac{d p}{d s}= & \left(\frac{d p}{d y}\right)_{x=0} \\
= & \frac{Q \mu}{2 a k h}\left[\operatorname{coth} \frac{\pi y}{a}+\sum_{1}^{\infty}(-1)^{m}\right. \\
& \left.\times\left\{\operatorname{coth} \frac{\pi(y-m d)}{a}+\operatorname{coth} \frac{\pi(y+m d)}{a}\right\}\right]
\end{aligned}
$$

According to Muskat, the neglect of the series terms beyond the first will lead to maximum errors of $0.4 \%$ for $\left.d / a \geqq 1 / 2^{1,},{ }^{3,}, 5\right)$. The equation of sweep efficiency for $d / a>1 / 2$ is given by; *

$$
\begin{aligned}
& p^{\prime}(x, y)=\frac{\mu Q}{2 \pi k h}\left[\ln \left(\cosh \begin{array}{c}
2 \pi y \\
a
\end{array}-\cos \begin{array}{c}
2 \pi x \\
a
\end{array}\right)\right.
\end{aligned}
$$

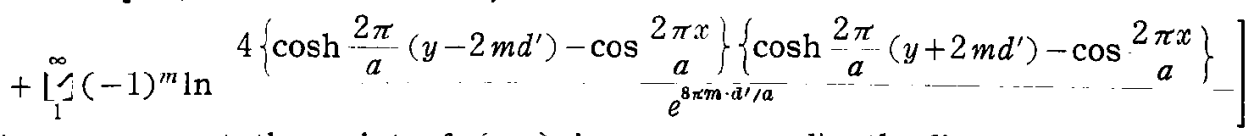

where $p^{\prime}$; pressure at the point of $(x, y)$ in the line drive to wells in a line.

The shortest-trave-time flowline is that along the $y$-axis from $d^{\prime}$ to 0 .

$$
\begin{aligned}
\frac{d p^{\prime}}{d s} & =\left(\frac{d p^{\prime}}{d y}\right)_{x=0} \\
& =\frac{\mu Q}{a k h}\left[\operatorname{coth} \frac{\pi y}{a}+\sum_{1}^{\infty}(-1)^{m}\right. \\
& \left.\times\left\{\operatorname{coth} \frac{\pi}{a}\left(y-2 m d^{\prime}\right)+\operatorname{coth} \frac{\pi}{a}\left(y-2 m d^{\prime}\right)\right\}\right]
\end{aligned}
$$

$$
* E=\frac{\left(\cosh ^{2} \frac{\pi d}{a} \ln \cosh \frac{\pi d}{a}-0.6932 \sinh ^{2}-\frac{\pi d}{a}\right)}{\left(\cosh ^{2} \frac{\pi}{a}-2\right) \frac{d}{a}}
$$

For the model of this study $d / a=9 / 7$

$$
E=0.658=65.8 \%
$$

2. A Line Drive to Wells in a Line

Procedures similar to Muskat's method for a direct-line-drive well network can be applied to obtain a sweep efficiency of a line drive to wells in a line.

Consider a unit of a dircet-line-drive well network. If the sizes of bore holes of injection and producing wells are the same, the pressure distribution will be symmetrical about $y=d / 2$ and the potential on the line, $y=d / 2$, will be $\left(P_{w}+P_{i}\right) / 2$, and $P_{i} / 2$ if $P_{w}=0$.

where $P_{w}$; pressure of producing well

$P_{i} ;$ pressure of injection well

$d$; distance between the lines of injection and producing wells

The pressure distribution for a line drive to wells in a line can be expressed by the following equation, if $p^{\prime}(x, y) / 2$ is inserted for $p(x, y)$ and $2 d^{\prime}$ for $d$ in equation (3-2). $\triangle$ In the case of the model used in this study $d^{\prime} / a=9 / 7$

All terms of the series are negligible and the equation $(3-7)$ reduces to

$$
\left(\frac{d p}{d y}\right)_{x=0}=\frac{Q \mu(\operatorname{coth} \pi y / a)}{a k h}
$$

Substituting (3-8) into (3-3)

$$
E={ }_{k h A}^{\mu Q} \cdot \underset{Q u}{a k h} \cdot \int_{d^{\prime}}^{0} \tanh \frac{\pi y}{a} d y
$$




$$
\begin{aligned}
& A=a d^{\prime} \\
& E=\left\{a_{i}^{\prime}\left(\pi d^{\prime}\right)\right\} \ln \left(\cosh \pi d^{\prime} / a\right)
\end{aligned}
$$

\section{Comparison of Calculation Methods}

For comparison, sweep efficiencies obtained by the three different calculation methods are shown here. The results by the analytical method do not contain experimental error, but a maximum error of $0.05 \%$ results from neglecting the series terms. To check the reproduciblity of this experiment, two runs of potential measurement for line drive were made, and sweep efficiencies for each flow net were calculated. The sweep efficiency for the first run was $83.2 \%$ and for the second run $82.3 \%$ as obtained by numerical integration method.

Table 2 Sweep efficiencies obtained by the three

\begin{tabular}{|c|c|c|c|}
\hline \multirow{2}{*}{$\begin{array}{l}\text { Calculation } \\
\text { method }\end{array}$} & \multicolumn{3}{|c|}{ Sweep efficiencies } \\
\hline & Direct-line-drive & Line $d$ & rive \\
\hline Numerical & 0.648 & $\begin{array}{l}\text { (1 st run) } \\
(2 \text { nd run })\end{array}$ & $\begin{array}{l}0.832 \\
0.823\end{array}$ \\
\hline Graphical & & & 0.830 \\
\hline Analytical & 0.658 & & 0.828 \\
\hline
\end{tabular}
different calculation methods

Comparing those results, errors of sweep efficiencies obtained by the numerical integration method, including experimental and calculation errors, are considered to be confined within $1 \%$.

In the following study of sweep efficiencies for the various cases of faulting, the numerical integration method was used because of its simplicity.

\section{Sweep Efficiencies of Impermeable Fault System}

\section{A. Faults Perpendicular to Direction of Edge-} water Approach

Faults extending perpendicularly to the flow of edge-water influx and at the midpoint between the source and a producing well, were first studied. Isopotentials were measured for cases of three different fault lengths. The flowlines were drawn in at suitable spacings, crossing at right angles to the isopotentials, to permit calculation of the times required for travel of particle on the flowlines. The poisitions of the front on flowlines were obtained when the front on the shortest-travel-time flowline first reached the electrode.

Fig. 9 shows the calculated flood fronts for edge water encroachment at breakthrough for the systems described above. Sweep efficiencies are summarized in Table 3 .

Table 3 Sweep efficiencies of faulted system

\begin{tabular}{c|c:c}
\hline Curve No. shown in & Length of fault & Sweep efficiency \\
Fig. 9 & 0 & $83.2 \%$ \\
I & $0.3 a$ & $73.1 \%$ \\
II & $0.585 a$ & $59.1 \%$ \\
III & $0.729 a$ & $52.1 \%$ \\
IV & & \\
\hline
\end{tabular}

The relation between fault length and areal sweep efficiency is shown graphically in Fig. 10. It is obvious that the sweep efficiency would be reduced to zero if the fault completely screened the flow. The curve shows that sweep efficiency decrease almost linearly from $83.2 \%$

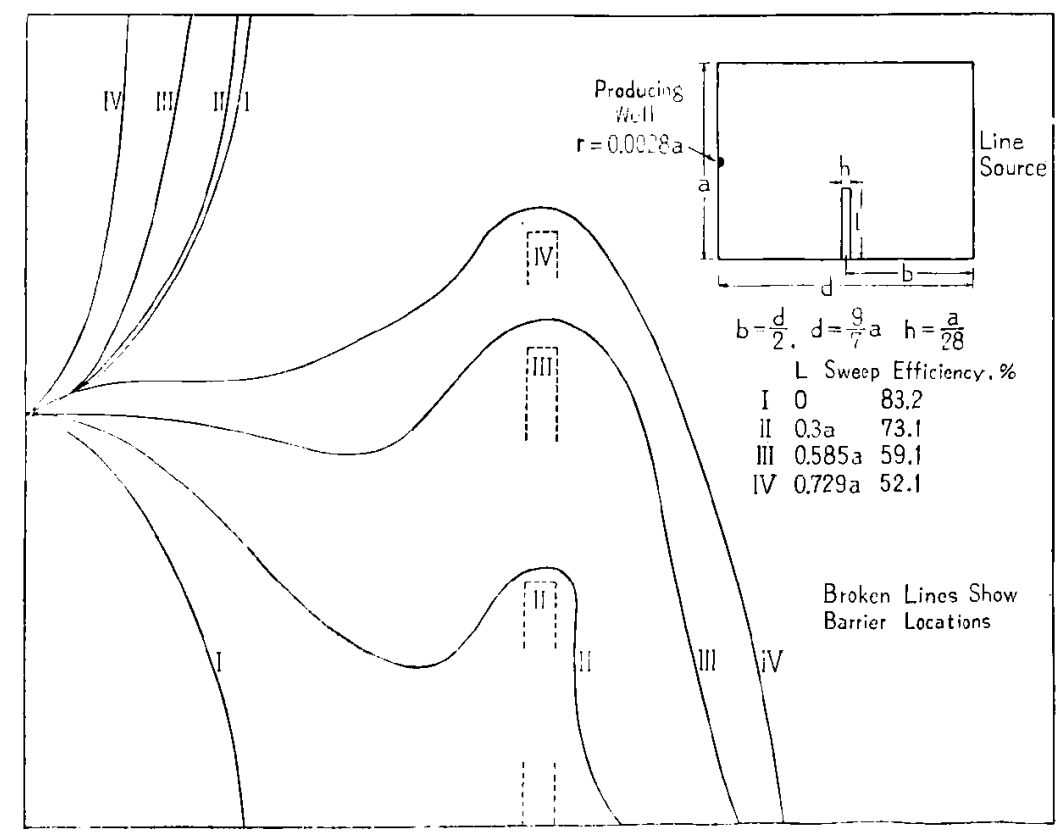

Fig. 9 Flood Fronts 


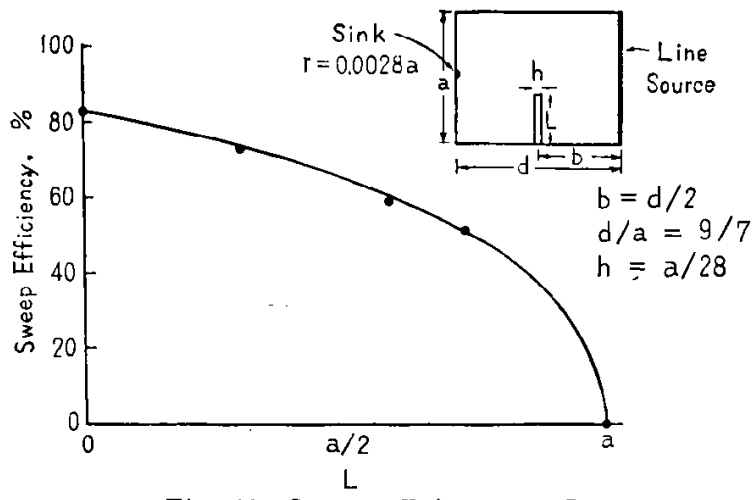

Fig. 10 Sweep efficiency vs $L$

to $52.1 \%$ with change of fault length from zero to $0.729 a$. At fault length, $(7 / 8) a$, or in the case of one-eighth of the width left open for fluid flow, the sweep efficiency is estimated to be one-half that for a non-faulted system. Above (7/8) $a$ of fault length, the sweep efficiency rapidly decreases to zero at $L=a$.

$B$. Sweep efficiency of System Including Faults Perpendicular to Direction of Flood, as Functions of Location between Injection and Producing Wells and Length

The systems studied were three different fault lengths located $1 / 6 \mathrm{th}, 3.2 / 9$ ths, $1 / 2 \mathrm{th}, 5.8 /$ 9 ths, and $5 / 6$ ths of the distance between input and output electrodes. Relations of sweep efficiency versus fault length at their several positions are tabulated in Table 4. Extrapolating and interpolating these data, Fig. 11 was obtained. Comparing the results, it will be noted that the minimum sweep efficiency of the constant length fault system is found at $b=d / 3$. It is to be noted that a better efficiency can be

Table 4 Sweep efficiencies

\begin{tabular}{c|c|c}
\hline & $\begin{array}{c}\text { Length of fault } \\
L\end{array}$ & $\begin{array}{c}\text { Sweep efficiency } \\
\%\end{array}$ \\
\hline \multirow{3}{*}{$b=d / 6$} & 0 & 64.8 \\
& $0.296 \mathrm{a}$ & 60.8 \\
& $0.586 \mathrm{a}$ & 53.7 \\
\hline & 0 & 64.8 \\
& $0.300 \mathrm{a}$ & 57.5 \\
& $0.586 \mathrm{a}$ & 47.4 \\
& 0 & -64.8 \\
& $0.189 \mathrm{a}$ & 62.1 \\
& $0.311 \mathrm{a}$ & 58.1 \\
& $0.593 \mathrm{a}$ & 52.9 \\
& $0.743 \mathrm{a}$ & 45.5 \\
\hline $5=5.8 d / 2$ & 0 & 64.8 \\
& $0.300 \mathrm{a}$ & 58.0 \\
& $0.586 \mathrm{a}$ & 52.8 \\
& $0.865 \mathrm{a}$ & 41.0 \\
\hline \multirow{3}{*}{$b=5 d / 6$} & 0 & 64.8 \\
& $0.296 \mathrm{a}$ & 61.1 \\
& $0.586 \mathrm{a}$ & 59.5 \\
\hline
\end{tabular}

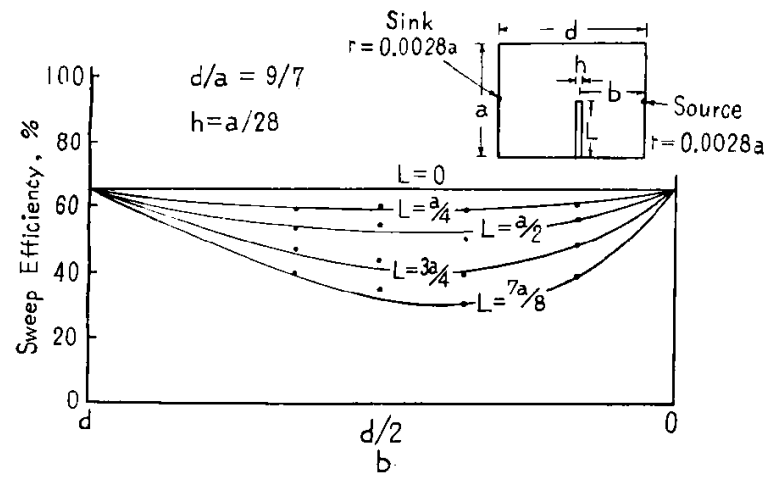

Fig. 11 Sweep efficiency vs $b$

expected by applying the flooding direction such that the area on the injection side separated by a fault is broader than that on the producing side. Better efficiencies at $b=(d-x)$ than those at $b=x$, where $x$ is less than $d / 2$, are found.

C. Sweep Efficiency of System Including Faults at Angles to the Direction of the Flood

Sweep efficiency is plotted as a function of fault angle to direction of flood on Fig. 12. It will be noted that a minimum efficiency is not found at $\theta=\pi / 2$, where the projected area or length to direction of flood is the largest, but at $\theta=\pi / 3$. Better efficiencies at $\theta=(\pi-x)$ than those at $\theta=x$, where $x$ is less than $\pi / 2$, are found. It can again be said that the flooding direction such that the area on the injection side separated by a fault is larger than that on the producing side, will result in better efficiency.

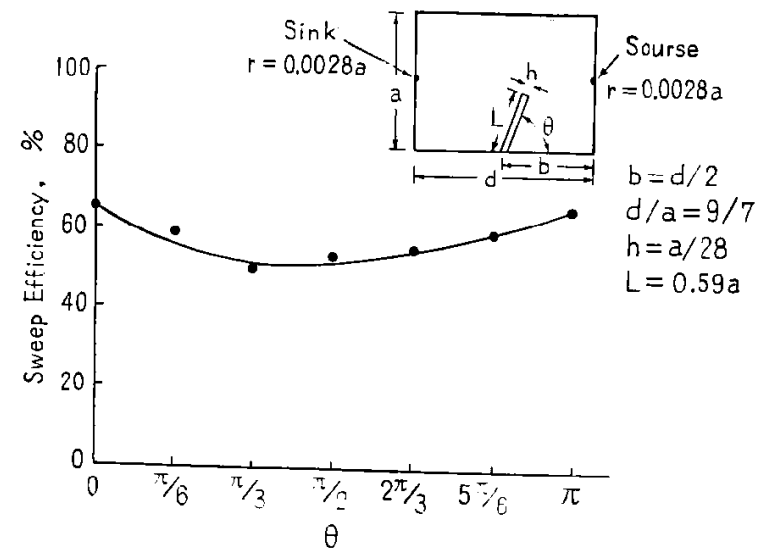

Fig. 12 Sweep efficiency vs $\theta$

\section{Sweep Efficiencies of Semi-Permeable Fault System}

Many faults are considered as semi-permable to fluid flow and are not complete barriers, as was assumed in the preceding analysis. The following studies were made for faults having some magnitude of permeability and perpen- 
dicular to the direction of the flood at the midpoint between injection and producing wells. In using the model to represent a semi-permeable fault system, non-conductive barriers were perforated with $7 / 32$ in. diameter holes. The ratio of summation of the area of the holes to total strip area is considered as the permeability ratios of 0.088 and 0.187 were studied.

The sweep efficiencies are plotted as functions of permeability ratios of fault to medium $k_{2} / k_{1}$, or resistivity ratios of medium to fault $R_{1} / R_{2}$, on Fig. 13. It is obvious that the sweep efficiency of the system, $k_{2} / k_{1}=1$, would be the same as that of no faulting system, and also that of $k_{2} / k_{1}=0$, would be the same as that of mpermeable faulting system as studied before.

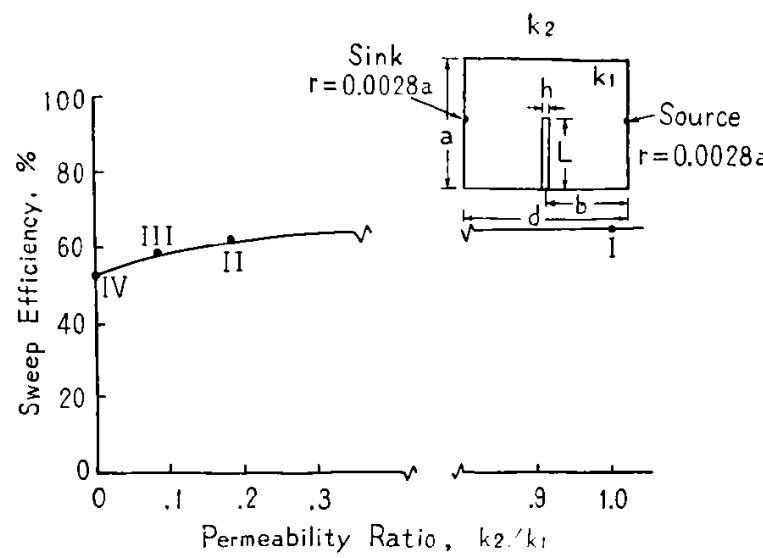

Fig. 13 Sweep efficiency vs Permeability ratio

Although only one fault length and one fault position with respect to input and output electrodes were studied, the same effects would be expected for other conditions. The sweep efficiency for a semi-permeable faulting system is in the intermediate range between those for a non-faulted system and an impermeable faulting system at a constant fault length. According as fault length becomes longer, the difference of sweep efficiencies for non-faulted and impermeable faulting systems is larger. Therefore, in general the longer the fault, the lower is the sweep efficiency; the longer the the greater would the effect of permeability ratio of fault to medium be expected.

\section{Conclusion}

The existence of faults in a flooding network, impermeable or semi-permeable, reduces the sweep efficiency of the pattern. The sweep efficiency depends on the length, position and orientation of the faulting system with respect to the direction of the flood.
It was found that the sweep efficiency may be reduced to one half or more when the impermeable faults, whose lengths exceed $7 / 8$ of the well spacing, extend perpendicular to the direction of the flood. For constant fault length, for faulting perpendicular to the flooding direction, the sweep efficiency is a minimum at $b=0.3 d$, where $b$ is the distance between faults and injection well, and $d$ is the spacing from the injection well to the producing well. Where faults are at angles to the direction of flood, the sweep efficiency is a minimum at $\theta=(1 / 3) \pi$, where $\theta$ is the angle between the fault and the flood direction.

For semi-permeable faulting systems, the ratio of the permeability of the fault to that of the medium becomes another factor controlling sweep efficiencies. The sweep efficiency for a semi-permeable faulting system is larger than that for an impermeable faulting system at a constant fault length, and less than that for a non-faulted system. The longer the fault, the greater would be the effect of permeability ratio on sweep efficiency.

The generalization of this study to actual oil field is concluded as following. The sweep efficiency of the pattern can be expected to be above one-half of the efficiency of non-faulted system, as long as the fault length does not exceed $85 \%$ of the well spacing, at the conventional well spacing. If the faults, impermeable or permeable, extend in length up to one-half the spacing, sweep efficiencies above $80 \%$ of those for non-faulted systems can be expected.

Where injection and producing wells must be partly separated by faults, a greater sweep efficiency will be obtained when the injection well is located such that the area between the injection well and the fault is greater than the area between the producing well and the fault.

If a favorable pattern or direction of flood can not be taken because of other reasons, further model studies at the given condition may provide useful information.

\section{Acknowledgement}

The author wishes to express his sincere appreciation to Professors J.A. Putnam, W.H. Somerton and J.F. Evernden of the University of California, Berkeley, California, for their guidance, constructive criticism and suggestions. He further wishes to thank Robert Darnaby for valuable discussion and arrangement for the experimental work. 


\section{Reference}

1) M. Muskat : Physical Principles Oil Production, McGraw-Hill Book Co., New York (1949).

2) W. L. Horner and W. A. Bruce: "Electrical Model Study of Secondary Recovery "; Secondary Recovery of Oil in the United States; American Petroleum Institute, 196 (1950)

3) M. Muskat: The Flow of Homogeneous Fluids Through Porous Media, McGraw-Hill Book Co., New York (1957).

4) G.H. Faucher: "Theoretical Consideration Involved in Secondary Recovery Operation"; Secondary Recovery of Oil in the United States; American Petroleum Institute, 184 (1950).

5) M. Muskat : Petrol. Tech.; 11. (6); American Institute of Mining and Metallurgical Engineers, Technical Publication No. 2490 (1948).

6) W. Hurst : Petrol. Eng. 25, (4), 538 (1953).

7) W. A. Bruce: Petrol. Tech. 6, (1); American Institute of Mining and Metallurgical Engineers.
Technical Publication No. 1550 (1943).

8) P.B. Grawford : "Estimated Effect of Vertical Fractures on Secondary Recovery" ; J. Petrol. Tech. 17, American Institute of Mining and Metallurgical Engineers, Technical Publication No. 3879 (1954).

9) W. A. Bruce: "Pressure Prediction for Oil Reservoir"; Petrol. Tech. 5, (2); American Institute of Mining and Metallurgical Engineers, Technical Publication No. 1454 (1942)

10) B. D. Lee: "Potentiometric Model Studies of Fluid Flow in Petroleum Reservoirs"; Petrol. Tech. 10, (5); American Institute of Mining and Metallurgical Engineers, Technical Publication No. 2262 (1947).

11) M.K. Hubbert : "Entrapment of Petroleum under Hydrodynamic Condition"; American Association of Petroleum Geologists; 37. (8), 1970 (1953).

12) M. K. Hubbert: "The theory of ground water motion": J. Geology, 48, (8), 844 (1940). 